\title{
Eduardo Carreño (Avilés, 13-10-1818 - París, 20-02-1841). Su herbario español recuperado
}

\author{
María A. Carrasco ${ }^{1}$ y Domingo Perea ${ }^{2}$
}

\begin{abstract}
Resumen: Carrasco, M. A. \& Perea, D. 2012. Eduardo Carreño (Avilés, 13-10-1818 - París, 20-02-1841). Su herbario español recuperado. Bot. Complut. 36: 123-130.

Presentamos la colección de plantas españolas procedentes de las herborizaciones de Eduardo Carreño que se encuentran en el herbario del Real Colegio Alfonso XII de San Lorenzo de El Escorial; todas ellas fueron recolectadas en España antes de su traslado a París en 1838. También incluímos un pliego de Festuca rubra Lag., que procede del herbario Lagasca y por tanto fue identificada por el autor del taxón. Esta colección, además de su interés histórico, tiene el valor añadido de ser las únicas plantas que se conservan de Carreño. Tambien se autentifica la letra de Eduardo Carreño y se dirime la cuestión de los destinatarios de su legado testamentario.
\end{abstract}

Palabras clave: colecciones históricas, Eduardo Carreño.

Abstract: Carrasco, M. A. \& Perea, D. 2012. Eduardo Carreño (Avilés, 13-10-1818 - París, 20-02-1841). His Spanish recovered herbaria. Bot. Complut. 36: 123-130.

We introduced a brief description of one set of plants found in the herbarium of the Real Colegio Alfonso XII of San Lorenzo de El Escorial. All of them were collected in Spain by Eduardo Carreño before moving to Paris in 1838. We also include one sheet of Festuca rubra Lag., belonging to Lagasca's herbaria, therefore was named by its discoverer. Other than the historical importance of these sheets, these are the only preserved plants collected by Carreño in Spain, increasing its historical value. Furthermore, we authenticate the handwriting of Carreño and we annul the current beliefs of who the real beneficiary of his will were.

Key words: historical collections, Eduardo Carreño.

\section{INTRODUCCIÓN}

Eduardo Carreño, discípulo predilecto de Mariano Lagasca, desarrolló su corta vida profesional primero en Madrid y después en París donde marchó el 27 de septiembre de 1838. La muerte de Lagasca unos meses después, en junio de 1839, convirtió a Mariano de la Paz Graells, entonces Director del Museo Nacional de Ciencias Naturales, en el interlocutor de Carreño en España; ambos se escribieron a menudo y las cartas se conservan en el archivo del Museo Nacional de Ciencias Naturales.

La temprana muerte de Carreño conmocionó a la comunidad botánica de la época de forma inusual para una persona que aún no había cumplido 23 años pero ya se perfilaba como la gran promesa española para la ciencia de las plantas. Cuando murió, se publicaron necrológicas y obituarios en las revistas científias y en los periódicos, y su biografía, actividad profesional y relaciones con los más preclaros botánicos de su época están recogidas en Colmeiro (1858: 204-205). A pesar del tiempo transcurrido su personalidad científica continúa despertando interés y se siguen publicando biografías y apuntes sobre él, destacando entre las últimas la de Baragaño (2010).

La relación de plantas procedentes de su herbario fueron enumeradas en Carrasco et al. (2001), especificando el número de pliegos que forman cada exsiccata. Ahora presentamos las que recogió en España; hemos tenido ocasión de estudiar estos materiales para reconstruir la actividad botánica de Eduardo Carreño por las etiquetas de los pliegos. Son colecciones con interés histórico y, que sepamos, las únicas que se conservan de este botánico.

\section{MATERIALES Y MÉTODOS}

El herbario de Eduardo Carreño se encuentra en los fondos del Real Colegio Alfonso XII de San Lorenzo de El Esco-

\footnotetext{
${ }^{1}$ General Pardiñas 118, E-28006 Madrid, España. carrascosalazar@telefonica.net

2 Real Colegio Alfonso XII, E-28200 San Lorenzo de El Escorial, Madrid, España.jeseso@colalfonsoxii.com

Recibido: 3 diciembre 2011. Aceptado: 2 febrero 2012.
} 
rial, que llamamos herbario RCAXII desde que empezamos a estudiarlo (Carrasco et al. 1996). Los materiales que recogió en España son 60 pliegos, casi todos de plantas vasculares; 5 de ellos, que estaban sin identificar, lo han sido por nosotros para presentarlos en este trabajo. Las plantas están bien preparadas y han aparecido en un paquete muy bien conservadas (Fig. 1); han sido desinfectadas, montadas sobre cartulina blanca y están etiquetadas (m. Carreño); las etiquetas son muy pobres (Fig. 2), aportan muy pocos datos, a veces unicamente localidad y fecha de recolección, y en ocasiones estos datos son muy poco precisos (por ejemplo «in montibus Asturicensis») y en todos los pliegos identificados, excepto en uno, que se indica, hay dos etiquetas, ambas manuscritas (m. Eduardo Carreño), una con el nombre del taxón y otra con el resto de la información, que generalmente consiste sólo en localidad y fecha. El pliego RCAXII 8854, que estaba sin identificar, aporta una somera descripción en latín. La abreviatura que aparece repetidamente: Jr. Bo. Ma., corresponde a Jardín Botánico de Madrid.

Hemos recurrido a las cartas de Carreño que conserva el Archivo del Museo Nacional de Ciencias Naturales para autentificar la letra de las etiquetas y también para aclarar a quien legó por testamento sus colecciones. Citamos las etiquetas literalmente, respetando la grafía original tanto ortográfica como de puntuación para los nombres de las plantas y las localidades, siguiendo las convenciones tipográficas propuestas por Burdet et al. (1979: 66). La estandarización de los datos del catálogo se realiza con las publicaciones siguientes: Brummitt et Powell

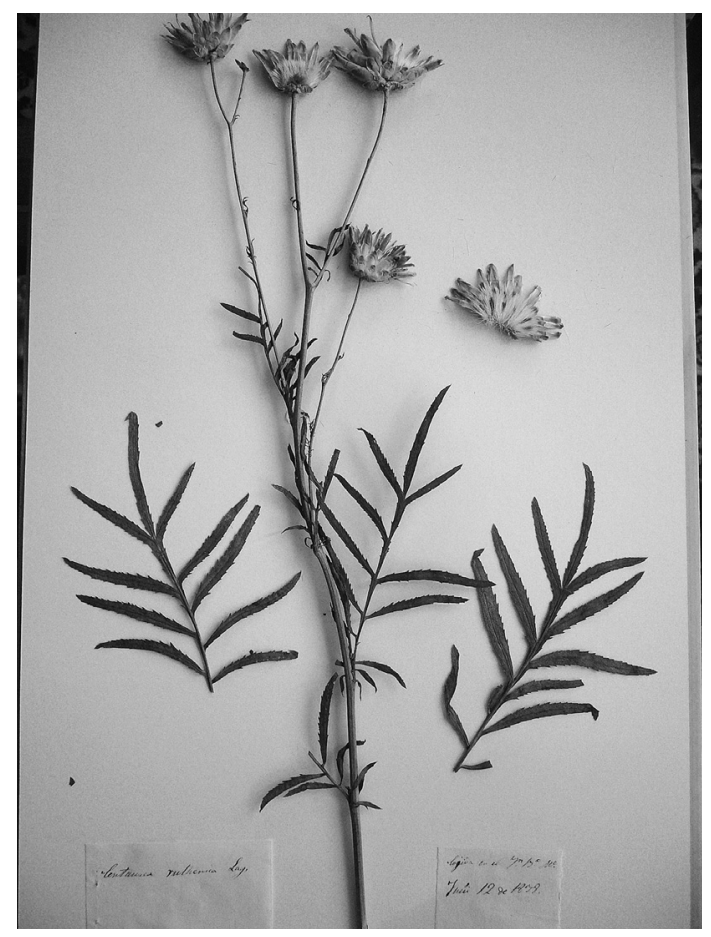

Fig. 1- Estado de conservación de las plantas.

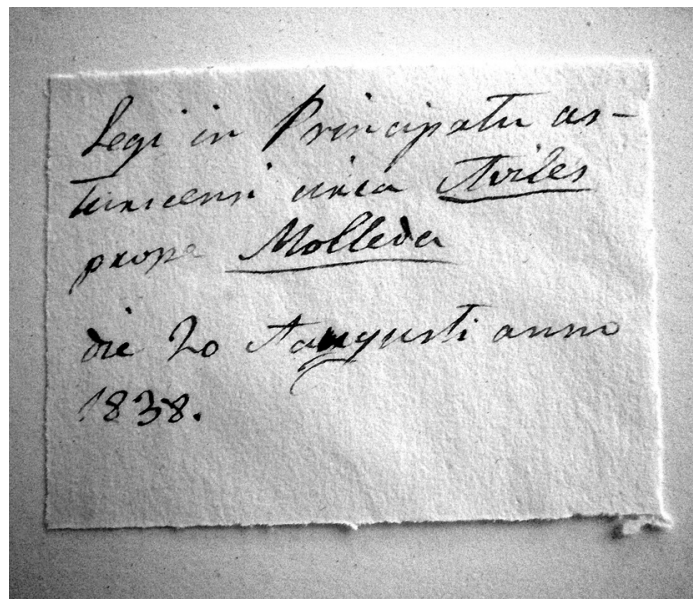

Fig. 2- Etiquetado de los pliegos (m. Eduardo Carreño).

(1992) para los autores de las especies y Stafleu et Cowan (1976) para la cita de las obras.

Las plantas se presentan en orden cronológico, separadas por años de recolección, y dentro de cada año, por fechas (si existen); si no tienen fecha seguimos el orden alfabético de especies para facilitar su consulta. Dada la corta vida que tuvo Carreño, son pocas las plantas recogidas cada año y por tanto fácil su localización en el catálogo. Nuestros comentarios, si ha lugar, se incluyen como observaciones detrás de cada taxón.

Presentamos un esquema taxonómico con los nombres de las plantas estudiadas, que aparecen numeradas y por orden alfabético, presentando en primer lugar los briófitos. Los nombres que consideramos válidos se escriben en letra negrita y son los que se ajustan a la estandarización de datos a la que aludimos anteriormente, dado que en los catálogos respetamos incluso las incorrecciones evidentes que a veces tienen las etiquetas originales. Si el nombre utilizado en las exsiccatas no es el actualmente considerado correcto lo escribimos en letra cursiva, seguido por el nombre válido en negrita una línea por debajo.

El acrónimo del herbario Real Colegio Alfonso XII (RCAXII) seguido del número de registro identifica cada pliego; esta designación de herbario es provisional ya que aún no está incluído en el listado de herbarios (http://sciweb.nybg.org/science2/ IndexHerbariorum.asp).

\section{RESULTADOS}

La letra de Carreño no aparece recogida en la relación de Burdet (1973), tampoco el archivo del Real Jardín Botánico de Madrid guarda ningún documento con su letra ni el herbario del Real Jardín Botánico de Madrid (MA) conserva ninguna planta recogida por Carreño; unicamente el pliego (MA 397547), procedente del Herbario Caroli Pau Herbarium Hispanicum, lleva una etiqueta sin fe- 


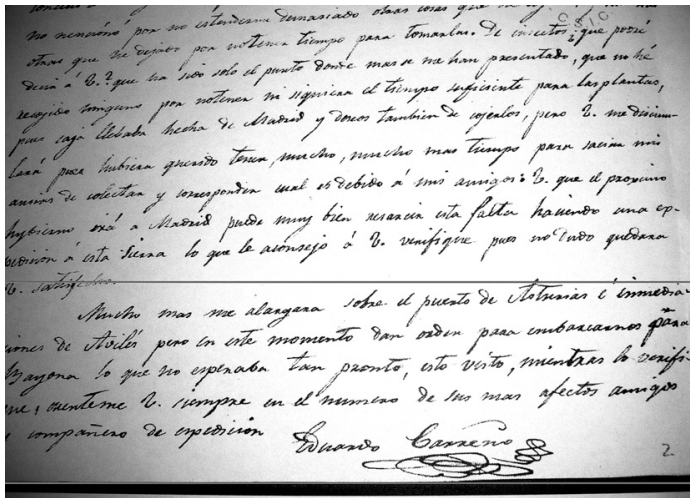

Fig. 3-Carta de Eduardo Carreño a Graells fechada el 31 de octubre de 1838.

cha ni identificación, sólo los datos: Asturias/ lg. Carreño?, pero manuscrita (m. Carlos Pau). El material ha sido identificado como Tilia platyphyllos por Carlos Aedo en fecha 4-XII-1990. Comparando la letra de las etiquetas que llevan los pliegos aparecidos en RCAXII con la de cartas manuscritas y firmadas por él que se conservan en el archivo del Museo Nacional de Ciencias Naturales, (ACN0349/004/001) (Fig. 2 y 3), podemos asegurar que la letra de las etiquetas de los pliegos que estudiamos pertenece a Eduardo Carreño.

Con respecto al destino que tuvieron las colecciones de Carreño tras su prematura muerte, hemos tenido ocasión de leer la carta que su padre, Pantaleón Carreño, escribió a Graells comunicandole la última voluntad de su hijo en relación a sus colecciones botánicas y zoológicas. Esta carta se conserva en el archivo del Museo Nacional de Ciencias Naturales (ACM0349/009/001) (Fig. 4).

Colmeiro (1858: 205) establece distintos destinatarios: el Museo Nacional de Ciencias Naturales para las zoológicas y a D. Mariano de la Paz Graells, por haber sido su maestro, le lega las botánicas. Este error, que transcribiendo a Colmeiro hemos repetido nosotros anteriormente (Carrasco et al., 2001), queda subsanado aquí con la presentación de la carta de Pantaleón Carreño cuyo párrafo en relación con el testamento de su hijo transcribimos: «.....que con autorización mía deseaba que su herbario y unas 4 ó 5 cajitas de minerales, como tambien una caja de insectos fuesen remitidas al Museo de Historia Natural de Madrid, para que se conservaran allí en su obsequio y memoria.....»; con esto se aclara definitivamente que todas las colecciones de Eduardo Carreño se legaron juntas y su destinatario fue el Museo de Historia Natural.

La repetida idea de que las colecciones botánicas le habían sido legadas a Graells por haber sido su maestro,

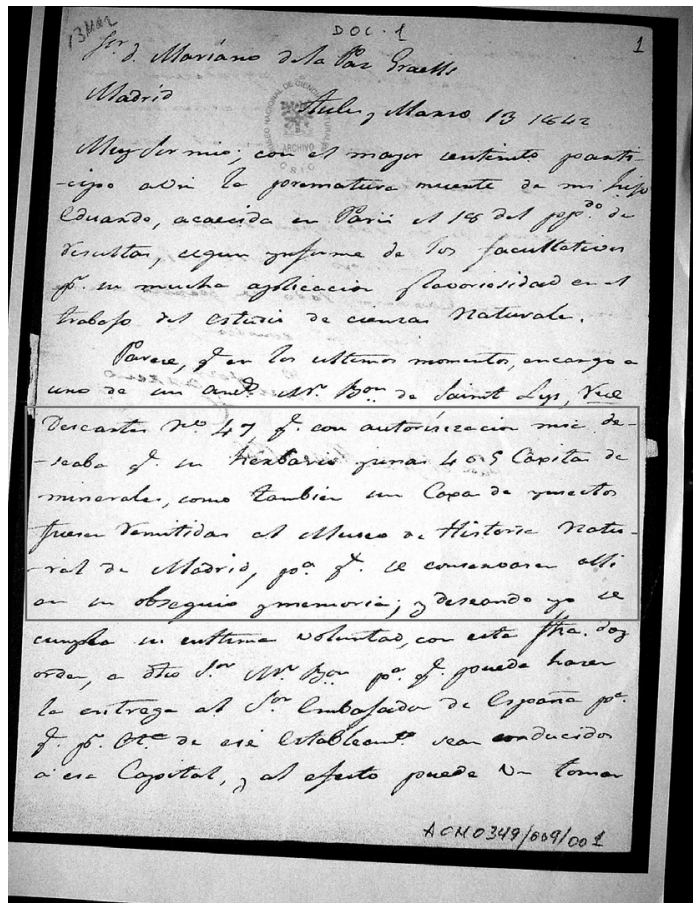

Fig. 4- Carta de Pantaleón Carreño a Graells fechada el 13 de marzo de 1842.

no se sustenta; en la correspondencia que mantuvo Carreño con Graells durante 1838, nunca se dirige a él llamandole «profesor» 0 «maestro», le saluda como «estimado amigo» y se despide como «su más afectuoso amigo y compañero de expedición» (ACM0349/009/001) (Fig. 3), o como «su afectísismo amigo y compañero de herborizaciones» (ACN0349/004/002) (Fig. 5).

Definitivamente, la razón por la que Graells recibe los materiales de Carreño es por su condición de director

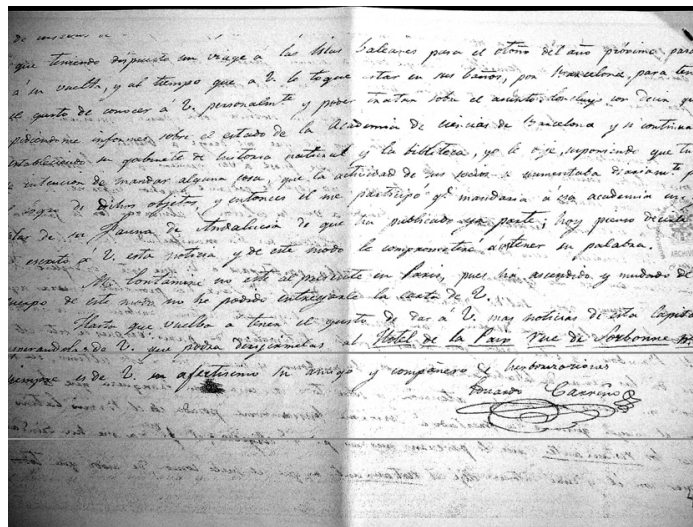

Fig. 5- Carta de Eduardo Carreño a Graells fechada el 27 de septiembre de 1838 . 
del Museo de Historia Natural de Madrid, que en ese momento incluía también el Real Jardín Botánico y sus colecciones.

Herbario español de Eduardo Carreño: plantas de Madrid y de Asturias. Las herborizaciones de Carreño se localizan en Madrid y sus alrededores, desde sus primeras recolecciones en el año 1835 hasta el 15 de julio de 1838 fecha en la que herboriza en la Sierra de Guadarrama. Después marcha a Asturias, a visitar a su familia en Avilés, antes de partir para establecerse en París. Dos días más tarde de sus recolecciones en Guadarrama, el 17 de julio de 1838, ya está en Asturias herborizando cerca de Avilés. En Asturias pasa algo más de dos meses y el 27 de septiembre embarca hacia Bayona desde Santander (Fig. 3). Las plantas que recogió en España durante estos años hasta su marcha a París son las que presentamos a continuación, ordenadas cronológicamente. Hemos procedido también a identificar los 5 pliegos que no lo estaban: dos plantas vasculares y 3 musgos. En el apartado de Observaciones de estos pliegos se indica: det. M. A. Carrasco.

\section{Año 1835}

Veronica digitata L. [RCAXII 8820]

Cercanias de Madrid. // Campos de Sn. Bernar /dino. // Mayo 16 de 1835.

Observaciones: especie que actualmente ha desaparecido de la provincia de Madrid (Martínez Ortega et al., 2009: 413-414). Debe llamarse Veronica chamaepithyoides Lam.

Cobea scandens Cav. [RCAXII 8844]

Mayo 31 de 1835 // Jn. Bo. Ma.

Festuca ciliata DC. [RCAXII 8856]

Cercanias de Madrid. // Junio 3 de 1835.

Observaciones: el nombre válido actual es Vulpia ciliata Dumort.

Plantago lanceolata L. [RCAXII 8849]

Cercanias de Madrid // Junio 8 de 1835.

Pelargonium acetosum Cav. / (Monad.) [RCAXII 8834] Agosto 20 de 1835 // Jn. Bo. Ma.

Observaciones: debe llamarse Pelargonium acetosum (L.) L'Hér.

Pelargonium alchemilloides Cav. / (Monad.) [RCAXII 8835]
Agosto 20 de 1835 // Jn. Bo. Ma.

Observaciones: debe llamarse Pelargonium alchemilloides (L.) L'Hér.

Poa verticillata Cav. [RCAXII 8814]

Cercanias de Madrid. // Orillas del Manzanares // Setbre. 1 de 1835.

Observaciones: debe llamarse Eragrostis pilosa (L.) P. Beauv.

Cyperus fuscus L. [RCAXII 8853]

Cercanias de Madrid. // Orillas del Manzanares. // Setbre. 4 de 1835.

\section{Año 1836}

Muscari comosum Mill. [RCAXII 8806]

(Dubi B.G.) // Hyacinthus comosus L.

Cercanias de Madrid. // Campos de Sn. Bernardino. // Mayo 17 de 1836.

Observaciones: debe llamarse Muscari comosum (L.) Mill.

Triticum ciliatum DC. var. / villosum Cav. [RCAXII 8818] Cercanias de Madrid / Cerro - negro. // Mayo 27 de 1836. Observaciones: debe llamarse Brachypodium distachyon (L.) Beauv. var. ciliatum (DC.) Beck.

Juncus glaucus Sm. [RCAXII 8809]

Cercanias de Madrid. // Pradera del Canal de / Manzanares. // Junio 7 de 1836.

Asclepias linaria Cav. [RCAXII 8845]

Julio 10 de 1836 // Jr. Bo. Ma.

Pascalia glauca Ort. Dec. [RCAXII 8840]

Agosto 28 de 1836 // Jn. Bo. Ma.

\section{Año 1837}

Teucrium capitatum L. [RCAXII 8826]

Cercanias de Madrid. / Cerro - negro. // Mayo 19 de 1837.

Festuca duriuscula L.? [RCAXII 8857]

Cercanias de Madrid. // Orilla del Manzanares. // Mayo 24 de 1837.

Silene porrigens Lag. [RCAXII 8833]

Junio 19 de 1837 // Jn. Bo. Ma.

Observaciones: debe llamarse Gypsophila pilosa Hudson 
Juncus communis Mey. var. effusus [RCAXII 8808]

Cercanias de Madrid pradera / de la Fuente de la Teja // Junio 27 de 1837.

Observaciones: debe llamarse Juncus effusus L.

Dichondra sericea Lag. [RCAXII 8842]

Agosto 4 de 1837 // Jn. Bo. Ma.

Observaciones: debe llamarse Dichondra sericea $\mathrm{Sw}$.

Solanum aeleagnifolium $\underline{\mathrm{Cav}}$. [RCAXII 8843]

Setbre. 1 de 1837 // Jn. Bo. Ma.

Cyperus acutiusculus Lag. [RCAXII 8831]

Setbre. 10 de $1837 / /$ Jn. Bo. Ma.

Scirpus annus All. [RCAXII 8832]

Octbre. 3 de 1837 // Jn. Bo. Ma.

Observaciones: debe llamarse Fimbristylis turkestanica (Regel.) B. Fedtsch.

\section{Año 1838}

Veronica triphyllos L. [RCAXII 8821]

Cercanias de Madrid. // Mayo 7 de 1838.

Ciclospermum ammi Lag. [RCAXII 8839]

Mayo 11 de 1838 // Jn. Bo. Ma.

Veronica hederaefolia L. [RCAXII 8830]

Cercanias de Madrid // Mayo 12 de 1838.

Observaciones: debe llamarse Veronica hederifolia L.

Koeleria calycina Perss. // Dantonia calycina Duf. [RCAXII 8855]

Cercanias de Madrid. // Mayo 27 de 1838.

Observaciones: debe llamarse Danthonia alpina Vest

Myosotis annua Moench. / (Dubi Bot. Gall.) [RCAXII 8800]

Cercanias de Madrid. // Orillas del Manzana / res. // Mayo 28 de 1838.

Observaciones: debe llamarse Myosotis arvensis (L.) Hill

Plantago lusitanica L. [RCAXII 8850]

Cercanias de Madrid // Retiro // Mayo 28 de 1838.

Observaciones: debe llamarse Plantago lagopus L.

Myosotis perennis Moench. / (Dubi Bot. Gall.) [RCAXII 8822]
Cercanias de Madrid. // Orillas del Manzanares // Mayo 30 de 1838.

Observaciones: debe llamarse Myosotis scorpioides L.

Rhinanthus hirsuta Lam. [RCAXII 8828]

Cercanias de Madrid. // Chamartin. // Mayo 31 de 1838.

Observaciones: debe llamarse Rhinanthus pumilus (Sterneck) Pau subsp. pumilus

Triticum Poa DC. [RCAXII 8817]

Cercanias de Madrid. // Chamartin // Mayo 31 de 1838.

Observaciones: debe llamarse Micropyrum tenellum (L.)

Link

Triticum Nardus DC. [RCAXII 8816]

Cercanias de Madrid. / / Retiro // Junio 4 de 1838.

Observaciones: debe llamarse Micropyrum tenellum (L.)

Link

Rottbollia ramosa Cav. [RCAXII 8851]

Cercanias de Madrid. // Junto al cuartel de Guard. / de corps. // Junio 13 de 1838.

Observaciones: debe llamarse Rottboellia ramosa Cav. y su nombre válido actual es Hainardia cylindrica (Willd.) Greuter

Poa eragrostis L. [RCAXII 8815]

Madrid. // Patio de la Escuela de / Medicina. // Junio 30 de 1838.

Observaciones: debe llamarse Eragrostis minor Host

Centaurea ruthenica Lag. [RCAXII 8837]

Cogida en el Jn. Bo. Ma. // Julii 12 de 1838.

Observaciones: el autor del taxón es Lam., no Lag., y su nombre válido actual es Rhaponticoides ruthenica (Lam.) M. V. Agab. \& Greuter, in Greuter (2003: 61).

\section{Briza maxima L. [RCAXII 8859]}

Legi in Castella veterum / in montibus Guadarrama // Die 15 Julii anno 1838.

\section{Pedicularis rostrata L.? [RCAXII 8827]}

Legi in montibus Guada / rramae. // Die 15 Julii anno 1838. Observaciones: debe llamarse Pedicularis kerneri Dalla Torre

Potamogeton natans L. / var. fluitans Roth. [RCAXII 8811] Legi in montibus / Guadarramae. // Die 15 Julii anno 1838. Observaciones: García Murillo (2010: 71), sigue el criterio de Kaplan (2005) quién eligió como neótipo para el 
nombre $P$. fluitans Roth el híbrido estéril entre $P$. lucens L. y P. natans L.

Veronica scutellata L. [RCAXII 8829]

Legi in montibus Gua / darramae. // Die 15 Julii anno 1838.

Linaria supina Desf. ? [RCAXII 8819]

Legi in Principatu astu/ricensis circa Gijon // die 17 Julii anno 1838.

Observaciones: se trata de Linaria supina (L.) Chaz.

Brunella grandiflora Moench. [RCAXII 8852]

Legi in montibus astu / ricensis circa Valganda // Die 19 Julii anno 1838.

Observaciones: debe llamarse Prunella grandiflora (L.) Scholler

Abama ossifraga DC. [RCAXII 8807]

Legi in montibus astu / ricensis circa Arvas. // Die $20 \mathrm{Ju}-$ lii anno 1838.

Observaciones: debe llamarse Narthecium ossifragum (L.) Huds.

Dicranum scoparium Hedw. [RCAXII 8805]

Legi in principatu asturi / censi prope Arvas. // Die $20 \mathrm{Ju}$ lii anno 1838.

Eriophorum angustifolium Reich. / (Bot. Gal.) [RCAXII 8813]

Legi in montibus As / turicensis. // Die 20 Julii anno 1838. Observaciones: debe nombrarse Eriophorum angustifolium Honck.

Luzula nivea Lam. [RCAXII 8810]

Legi in montibus astu / ricensis. // Die 20 Julii anno 1838. Observaciones: debe nombrarse Luzula nivea (L.) DC.

Lysimachia Numularia L. [RCAXII 8801]

Legi in Principatu as /turicensi circa Aviles / prope Molleda // die 20 Augusti anno / 1838.

Observaciones: debe nombrarse Lysimachia nummularia L. Aunque la especie, que aparentemente ha desaparecido de la flora española, era frecuente en el siglo XIX, Villar (1997: 51), los materiales recogidos por Carreño no pertenecen a esa especie. Deben atribuirse a Lysimachia nemorum L.

Polytrichum commune Hedw. [RCAXII 8858]

Legi in Principatu as / turicensi prope Arvas. // Die $20 \mathrm{Ju}$ lii anno 1838.
Observaciones: este material estaba sin nombre, ha sido det. M. A. Carrasco

Polytrichum juniperinum Hedw. [RCAXII 8802]

Legi in Principatu / asturicensi prope Pajares // Die $20 \mathrm{Ju}$ lii anno 1838.

Observaciones: este material estaba sin nombre, ha sido det. M. A. Carrasco

Tortula subulata L. [RCAXII 8804]

legi in Principatu as / turicensi prope Arvas // Die $20 \mathrm{Ju}-$ lii anno 1838.

Asplenium marinum L. [RCAXII 8823]

Legi in Principatu astu / ricensi in Aviles. // Die 30 Julii anno 1838.

Elymus gliciphyllus [RCAXII 8838]

(H.M) //Agosto 10 de 1838. // Jn. Bo. Ma.

Observaciones: se trata, sin duda, de un error de Carreño dado que el nombre Elymus gliciphyllus no ha sido nunca utilizado. Es Agropyron brachyphyllum Boiss. \& Hausskn. ex Boiss., Fl. Orient. 5 (2): 663 (1884), cuyo nombre válido actualmente es Elymus brachyphyllus (Boiss. \& Hausskn.) Ä Lowe, Feddes Repert. 95(7-8): 458. (1884).

Atrichum angustatum Hedw. [RCAXII 8803]

Legi in Principatu asturum / circa Aviles prope Molleda // Die 19 Augusti anno 1838.

Observaciones: este material estaba sin nombre, ha sido det. M. A. Carrasco

Isolepis cernua (Vahl) Roemer \& Schultes [RCAXII 8854] Culmo capilaceo foliis setaceis / Spica unica pauciflora, fructibus tricuetris subglobosis

Legi in principatu as / turiensi circa Aviles // Die $19 \mathrm{Au}-$ gustii anno / 1838.

Observaciones: este material, con dos etiquetas, una con una somera descripción latina y la otra con localidad y fecha, estaba sin nombre, ha sido det. M. A. Carrasco

Blechnum spicant (L.) Roth var. spicant [RCAXII 8824] Legi in Principatu astu / ricensi circa Aviles. // Die $20 \mathrm{Au}$ gusti anno 1838.

Observaciones: este material estaba sin nombre, ha sido det. M. A. Carrasco

Neottia spiralis Sw. // Ophrys spiralis L. [RCAXII 8825] Legi circa Santander // Die 22 Setbre. anno 1838.

Observaciones: debe llamarse Spiranthes spiralis (L.) Chevall. 
Maurandia personata Lag. [RCAXII 8841]

Novbre. 10 de 1838 // Jn. Bo. Ma.

Observaciones: esta etiqueta contiene un error en la fecha. La planta estaba empaquetada con todas las demás y la letra es, sin duda, de Carreño, pero no pudo ser recogida por él ya que embarcó hacia Bayona el 27 de septiembre, como atestigua la carta que con esa fecha escribió a Graells (Fig. 3), y no existe ningún testimonio de que regresase a España.

\section{Sin fecha de recolección}

Carex divisa Huds. ? [RCAXII 8812]

Circa Matritum.

Dyneba arabica Lag. [RCAXII 8848]

Observaciones: debe llamarse Dinebra arabica Jacquin, Fragment. Bot: 77-79, Tabula 121, fig. 1 (1809).

Festuca scabra Lag. [RCAXII 8847]

Lagasca dedit. Ex / hervario (sic) suo.

Observaciones: debe llamarse Brachypodium mexicanum

(Roem. \& Schult.) Link.

Fontanesia phyllireoides La Billard [RCAXII 8846]

Observaciones: hay un error de escritura, debe llamarse Fontanesia phillyreoides Labill.

Hibiscus trionum (Cav.) [RCAXII 8836]

Setbre. Jard. Bot. Mat.

Observaciones: este pliego tiene una única etiqueta.

Esquema taxonómico: en el siguiente esquema recogemos los taxones citados ordenados alfabéticamente; primero los briofitos y después las plantas vasculares. Los nombres numerados son los que aparecen en las etiquetas, incluso en los casos de evidente error. Si los nombres están escritos erróneamente o no son actualmente válidos, se presentan en cursiva y con indicación del nombre actualizado en negrita (una línea por debajo del anterior).

\section{Briofitos}

1. Atrichum angustatum Hedw. [RCAXII 8803]

2. Dicranum scoparium Hedw. [RCAXII 8805]

3. Polytrichum commune Hedw. [RCAXII 8824]

4. Polytrichum juniperinum Hedw. [RCAXII 8802]

5. Tortula subulata L. [RCAXII 8804]

\section{Plantas vasculares}

6. Abama ossifraga DC. [RCAXII 8807]

Narthecium ossifragum (L.) Huds.
7. Asclepias linaria Cav. [RCAXII 8845]

8. Asplenium marinum L. [RCAXII 8823]

9. Blechnum spicant (L.) Roth var. spicant [RCAXII 8824]

10. Briza maxima L. [RCAXII 8859]

11. Brunella grandiflora Moench. [RCAXII 8852]

Prunella grandiflora (L.) Scholler

12. Carex divisa Huds. [RCAXII 8812]

13. Centaurea ruthenica Lag. [RCAXII 8837] Rhaponticoides ruthenica (Lam.) M. V. Agab. \& Greuter

14. Ciclospermum ammi Lag. [RCAXII 8839]

15. Cobea scandens Cav. [RCAXII 8844]

16. Cyperus acutiusculus Lag. [RCAXII 8831]

17. Cyperus fuscus L. [RCAXII 8853]

18. Koeleria calycina Perss. [RCAXII 8855] Danthonia alpina Vest

19. Dichondra sericea Lag. [RCAXII 8842] Dichondra sericea Sw.

20. Dyneba arabica Lag. [RCAXII 8848] Dinebra arabica Jacquin

21. Elymus gliciphyllus [RCAXII 8838] Elymus brachyphyllus (Boiss. \& Hausskn.) Ä Lowe

22. Eriophorum angustifolium Reich. [RCAXII 8813] Eriophorum angustifolium Honck.

23. Festuca ciliata DC. [RCAXII 8856] Vulpia ciliata Dumort.

24. Festuca duriuscula L. [RCAXII 8857] Festuca rubra L.

25. Festuca scabra Lag. [RCAXII 8847] Brachypodium mexicanum (Roem. \& Schult.) Link

26. Fontanesia phyllireoides La Billard [RCAXII 8846] Fontanesia phillyreoides Labill.

27. Hibiscus trionum Cav. [RCAXII 8836]

28. Isolepis cernua (Vahl) Roemer \& Schultes [RCAXII 8854]

29. Juncus glaucus Sm. [RCAXII 8809] Juncus inflexus L.

30. Juncus communis Mey. var. effusus [RCAXII 8808] Juncus effusus L.

31. Linaria supina Desf. ? [RCAXII 8819] Linaria supina (L.) Chaz.

32. Luzula nivea Lam. [RCAXII 8810] Luzula nivea (L.) DC.

33. Lysimachia Numularia L. [RCAXII 8801] Lysimachia nemorum L.

34. Maurandia personata Lag. [RCAXII 8841]

35. Muscari comosum Mill. [RCAXII 8806] Muscari comosum (L.) Mill.

36. Myosotis annua Moench. [RCAXII 8800] Myosotis arvensis (L.) Hill

37. Myosotis perennis Moench. [RCAXII8822] Myosotis scorpioides L.

38. Neottia spiralis Sw. // Ophrys spiralis L. [RCAXII 8825] Spiranthes spiralis (L.) Chevall.

39. Pascalia glauca Ortega [RCAXII 8840]

40. Pedicularis rostrata L.? [RCAXII 8827] Pedicularis kerneri Dalla Torre

41. Pelargonium acetosum Cav. [RCAXII 8834] Pelargonium acetosum (L.) L'Hér. 
42. Pelargonium alchemilloides Cav. [RCAXII 8835] Pelargonium alchemilloides (L.) L'Hér.

43. Plantago lanceolata L. [RCAXII 8849]

44. Plantago lusitanica L. [RCAXII 8850] Plantago lagopus L.

45. Poa eragrostis L. [RCAXII 8815] Eragrostis minor Host

46. Poa verticillata Cav. [RCAXII 8814] Eragrostis pilosa (L.) P. Beauv.

47. Potamogeton natans L. var. fluitans Roth. [RCAXII 8811] P. lucens L. X P. natans L.

48. Rhinanthus hirsuta Lam. [RCAXII 8828] Rhinanthus pumilus (Sterneck) Pau subsp. pumilus

49. Rottbollia ramosa Cav. Hainardia cylindrica (Willd.) Greuter

50. Scirpus annus All. [RCAXII 8832] Fimbristylis turkestanica (Regel.) B. Fedtsch.

51. Silene porrigens Lag. [RCAXII 8833] Gypsophila pilosa Hudson

52. Solanum aeleagnifolium Cav. [RCAXII 8843]

53. Teucrium capitatum L. [RCAXII 8826]

54. Triticum ciliatum DC. var. villosum Cav. [RCAXII 8818] Brachypodium distachyon (L.) Beauv. var. ciliatum (DC.) Beck.

55. Triticum Nardus DC. [RCAXII 8816] Micropyrum tenellum (L.) Link

56. Triticum Poa DC. [RCAXII 8817] Micropyrum tenellum (L.) Link

57. Veronica digitata L. [RCAXII 8820] Veronica chamaepithyoides Lam.

58. Veronica hederaefolia L. [RCAXII 8830] Veronica hederifolia L.

59. Veronica scutellata L. [RCAXII 8829]

60. Veronica triphyllos L. [RCAXII 8821]

\section{CONCLUSIONES}

Se han recuperado 60 pliegos de plantas españolas procedentes de recolecciones de Eduardo Carreño en España durante los años 1835 a 1838. Esta colección forma parte del herbario que Mariano de la Paz Graells depositó en el Real Colegio Alfonso XII de San Lorenzo de El Escorial y a su importancia histórica se añade el valor de ser las únicas plantas españolas que se conservan recogidas por Carreño; entre ellas, procedente del herbario de Lagasca, hay un pliego de Festuca rubra Lag. Anotese el herbario RCAXII como depositario de las exsiccatas de Carreño.

Se ha completado la identificación de los pliegos de esta exsiccata presentando también la actualización de los nombres de las especies. Se ha identificado la letra de Eduardo Carreño a partir de cartas manuscritas. Se aclara el error acerca del legado testamentario de Eduardo Carreño.

\section{AGRADECIMIENTOS}

Agradecemos sinceramente las facilidades que nos han ofrecido los responsables del Archivo del Museo Nacional de Ciencias Naturales en relación con el epistolario Carreño y los del Archivo y Herbario del Real Jardín Botánico de Madrid. y al Prof. Vicente Mazimpaka, de la Universidad Autónoma de Madrid, quien atendió amablemente nuestras consultas sobre los musgos de la colección Carreño.

Nuestra gratitud también para los doctores Velayos y Quintanar, del Real Jardín Botánico de Madrid, CSIC, y al profesor Mazimpaka, de la Universidad Autónoma de Madrid; la ayuda de todos ellos, solucionando los problemas nomenclaturales que les planteamos, ha sido inestimable.

\section{BIBLIOGRAFÍA}

BARAGAÑo, R. 2010. Comerciodigital.com el 09.01.10 - 03:09. Brummitt, R. K. \& Powell, C. 1992. Authors of plant names. Royal Botanic Gardens, Kew. London.

BuRDET, H. M. 1973. Cartulae ad botanicorum graphicem. III. Candollea 28: 407-440.

Burdet, H. M.; Charpin, A. \& JaCquemoud, F. 1979. Types nomenclaturaux des taxons décrits par Boissier, Leresche et Levier à la suite de leurs excursions en Espagne de 1878 et 1879. Mém. Soc. Bot. Genève 1: 63-82.

Carrasco, M. A.; Perea, D. \& García, A. 1996. Types of species decribed by M. P. Graells (1809-1898), found in the Real Colegio Alfonso XII (San Lorenzo de El Escorial, Madrid). Bot. Complut. 21: 87-97.

Carrasco, M. A.; Martín-Blanco, C. J. \& Perea, D. 2001. Herbaria of E. Carreño (1818-1841) and of M. P. Graells (18091898) discovered in the Real Colegio Alfonso XII of San Lorenzo de El Escorial, Madrid, Spain. Taxon 50: 587-591.

Colmeiro, M. 1858. La Botánica y los Botánicos de la Peninsula Hispano-Lusitana. Estudios Bibliográficos y Bio- gráficos. Imprenta y Estereotipia de M. Rivadeneyra. Madrid.

García Murillo, P. 2010. Potamogeton L. En S. Talavera, M. J. Gallego, C. Romero Zarco \& A. Herrero (Eds.), Flora iberica 17: 64-85.

Greuter, W. 2003. The Euro+Med treatment of Cardueae (Compositae) - generic concepts and new names. Willdenowia 33(1): 49-61.

Kaplan, Z. 2005. Neotypification of Potamogeton x fluitans Roth and the distribution of this hybrid. Taxon 54: 823-824.

Martínez Ortega, M. M.; SÁnchez Aguado, J. A. \& Rico, E. 2009. Veronica L. En C. Benedí, E. Rico, J. Güemes \& A. Herrero (Eds.), Flora iberica, 13: 360-434.

Stafleu, F. A. \& Cowan, R. S. 1976. Taxonomic literature, 1 (A-G), Regnum Vegetabile. Utrech.

Villar, L. 1997. Lysimachia L. En S. Castroviejo, C. Aedo, M. Laínz, R. Morales, F. Muñoz Garmendia, G. Nieto Feliner \& J. Paiva (Eds.), Flora iberica 5: 46-51. 